ISSN 1991-8631

Short Communication

http://indexmedicus.afro.who.int

\title{
Survenue des obstructions tubaires chez les femmes à Cotonou (Bénin) : rôle des bactéries
}

\author{
S. GANDJI ${ }^{1}$, H.S. BANKOLE ${ }^{2}$, T.V. DOUGNON ${ }^{1 *}$, J. DA SILVA ${ }^{3}$, C. ZANNOU ${ }^{3}$ et \\ O. BIAOU ${ }^{4}$ \\ ${ }^{I}$ Ecole Polytechnique d'Abomey-Calavi, Département de Génie d'Imagerie Médicale et de Radiologie (GIMR), \\ Université d'Abomey-Calavi, 01 BP 2009 Cotonou, Bénin. \\ ${ }^{2}$ Ecole Polytechnique d'Abomey-Calavi, Laboratoire de Recherche en Biologie Appliquée, Université \\ d'Abomey-Calavi, 01 BP 2009 Cotonou, Bénin. \\ ${ }^{3}$ Ecole Polytechnique d'Abomey-Calavi, Département de Génie de Biologie Humaine (GBH), Université \\ d'Abomey-Calavi, 01 BP 2009 Cotonou, Bénin. \\ ${ }^{4}$ Faculté des Sciences de la Santé, Université d'Abomey-Calavi, Champ de Foire, Cotonou, Bénin. \\ ${ }^{*}$ Corresponding author: E-mail: victorien88@hotmail.com; Tel.00 (229) 97736446.
}

\section{RESUME}

Les trompes utérines jouent un rôle très important dans la reproduction humaine. Elles peuvent être facilement atteintes par diverses pathologies dont l'obstruction tubaire. La présente étude a permis de déterminer le taux d'obstructions tubaires dues aux infections et de relever les bactéries en cause à Cotonou. Pour ce faire, 111 femmes venues pour hystérosalpingographie (HSG) ont été soumises aussi à des prélèvements sanguins et cervicaux (bilans microbiologiques). Au total, 51,3\% de femmes souffrent d'obstructions tubaires. La majorité d'entre elles $(89,4 \%)$ a un âge compris entre 25 et 39 ans. Les germes banaux, les mycoplasmes et Chlamydia trachomatis ont été isolés dans respectivement 28,1\%, 38,6\% et 36,8\% des cas. Les différents germes étudiés se retrouvent aussi bien chez les sujets souffrant d'obstructions tubaires que chez celles qui n'en souffrent pas. Les infections dues aux germes banaux, aux mycoplasmes et à $C$. trachomatis pourraient engendrer une obstruction tubaire, d'où l'intérêt d'un traitement correct de ces infections afin d'éviter qu'elles ne deviennent chroniques.

(C) 2013 International Formulae Group. All rights reserved.

Mots clés : Obstructions tubaires, Hystérosalpingographie, Infections.

\section{INTRODUCTION}

Tant chez l'homme que chez la femme, l'infertilité demeure un sujet de préoccupation majeure. Mais dans la plupart des sociétés et particulièrement en Afrique, lorsqu'un couple est confronté à ce problème, c'est souvent la femme qui est indexée. L'infertilité constitue un drame social et est souvent l'une des causes majeures de mésentente conjugale ou de divorce. En Afrique, un enfant consolide les liens qui existent entre les familles. L'infertilité constitue alors une rude épreuve que le couple doit surmonter. Il s'agit d'une situation qui nécessite un traitement long et 
coûteux. Elle est engendrée par de nombreux facteurs étiologiques, et de leurs connaissances précises, dépend la prise en charge adéquate des couples infertiles. L'infertilité touche un grand nombre de patientes en Afrique Noire et l'étiologie tubaire est souvent évoquée (Attolou et Hitoemeto, 2004). De nombreux travaux ont été effectués sur ce sujet dont ceux de N'dakena et al. (1993) qui avaient fait ressortir les aspects radiologiques de la pathologie tubaire dans la stérilité féminine au CHU de Lomé en 1993. De Muylder (1995) au Zimbabwe avait étudié la place de l'HSG dans l'évaluation d'une infertilité en Afrique Noire. Attolou et Hitoemeto (2004) avaient démontré que $16 \%$ des obstructions tubaires proximales étaient d'origine spasmodique. Quel peut donc être la part de responsabilité des microbes dans les obstructions tubaires? C'est la raison pour laquelle la présente étude a été initiée. Elle a eu pour but de déterminer le taux d'obstructions tubaires dues aux infections et surtout de préciser les microorganismes impliqués.

\section{MATERIEL ET METHODES Matériel d'étude}

Il est constitué aussi bien de matériel radiologique que microbiologique. Le matériel radiologique était constitué du cabaret à HSG contenant des spéculums, des pinces, des hystéromètres, des canules de Bommelaer, des seringues, des aspirateurs, des baladeuses, des antiseptiques, du lubrifiant, des antispasmodiques injectables, des cassettes et films $18 \mathrm{~cm}$ x $24 \mathrm{~cm}$ et celui microbiologique d'écouvillons et aiguilles stériles; d'eau physiologique, de bouillon Trypcase Soja entre autres.

\section{Méthodes d'étude}

Une étude prospective et transversale a été menée sur une population de femmes béninoises dans la période allant du 10 octobre 2006 au 29 mai 2007 à l'Hôpital de la Mère et de l'Enfant (HOMEL). Un échantillon de 111 patientes ayant bénéficié d'une HSG a été constitué. Des critères d'inclusion ont été définis: femme de race noire en âge de procréer et en période pré-ovulatoire; détention d'une demande d'examen de HSG ; suspicion d'obstructions tubaires. De même, les critères de non-inclusion (femme en période ovulatoire ou post-ovulatoire ; femme en métrorragie ; femmes gestantes ou femmes ménopausées ; femmes présentant une contreindication à l'HSG ont été définis. L'examen de HSG a été réalisé conformément à la méthodologie de Bourrit et Kinkel (2007). C'est un examen qui consiste en une radiographie aux rayons $\mathrm{X}$ de l'utérus et des trompes de Fallope après injection d'une substance opaque dans la cavité utérine.

Cinq (5) $\mathrm{ml}$ de sang ont été recueillis dans un tube sec stérile chez chaque patiente au niveau des veines du pli du coude. Les prélèvements cervicaux ont été faits après avoir introduit le spéculum dans le vagin. Un écouvillon stérile a été introduit dans la cavité fusiforme de l'endocol. Par frottement, des sécrétions endocervicales ont été ramenées. Les prélèvements ainsi obtenus ont été envoyés au service de laboratoire pour examens microbiologiques (Recherche de germes banaux, de mycoplasmes et de $C$. trachomatis). Toutes les informations obtenues sur les patientes ont été enregistrées sur des fiches d'enquête, lesquelles ont été classées et ordonnées pour un traitement informatique au moyen du logiciel Epi info 6.4 version française; ce qui a permis de calculer les moyennes et fréquences des différents paramètres ainsi que le risque relatif. L'étude a pris soin de recueillir le consentement de tous les sujets recensés dans l'anonymat.

\section{RESULTATS ET DISCUSSION}

Cette étude a permis de faire ressortir des aspects radiographiques et microbiologiques des obstructions tubaires et de préciser les germes impliqués. La moyenne d'âge de nos patientes est de 31 ans. Elle est assimilable à celle qu'avait observée Lahady et al. (2000) (30 ans) et à celle de Muteganya 
et al. (1997) (29,4 ans). Quatre-vingt-sept virgule trois pour cent $(87,3 \%)$ sont des femmes de la tranche d'âge de 25 à 39 ans (Figure 1). Cela prouve que c'est dans cette période que les femmes ont un désir de maternité beaucoup plus prononcé au Bénin. Godjo (1994) avait démontré que $67,3 \%$ de femmes stériles se retrouvaient dans la tranche de 20 à 34 ans. Vingt-trois patientes (23) sont des nullipares, soit un pourcentage de $20,7 \%$. Cela permet de déduire que la majorité (79,3\%) des patientes incluses étaient des femmes ayant porté au moins une grossesse, laquelle n'a pu aboutir, certainement à cause de raisons étiologiques.

Ces femmes présentent donc une infertilité secondaire qui pourrait être due soit à des infections post-abortum ou post-partum. Nos résultats corroborent ceux de N'dakena et al. (1993) qui avaient obtenu $75 \%$ de cas d'infertilités secondaires.

Sur 111 HSG réalisées, 51,3\% de femmes présentent une obstruction tubaire. Cette fréquence est proche de celle de Lahady et al. (2000) qui ont obtenu $61,29 \%$ mais diffère de celle obtenue par N'dakena et al. (1993) qui avaient trouvé un taux de $30 \%$ sur 1314 cas. Cette différence pourrait s'expliquer par la grande taille de l'échantillonnage et l'usage d'un antispasmodique dans certains cas. Les fréquences d'obstruction tubaire les plus élevées se retrouvent dans les tranches d'âge allant de 25 à 39 ans soit 89,4\%. En effet, elles constituent la majorité de la population étudiée et il est aisé de déduire que c'est dans ces tranches d'âge que se pose le plus le problème d'infertilité d'origine tubaire. Il n'y a pas eu une grande différence entre les fréquences des principaux types d'obstruction tubaire [proximale $(43,9 \%)$, distale $(38,6 \%)$ ]. Toutefois, nous notons une fréquence légèrement élevée dans les cas d'obstruction tubaire proximale. C'est aussi dans ces cas qu'on rencontre le plus les cas d'obstructions spasmodiques tels que l'ont décrit Palmer et al. (1975) puis Attolou et Hitoemeto (2004). Ces derniers avaient remarqué que sur 50 cas d'obstruction tubaire proximale, 8 soit $16 \%$ avaient été levées par l'injection d'un antispasmodique. Selon Palmer et al. (1975), la cause la plus fréquente d'un faux diagnostic d'obstruction tubaire proximale est certainement le spasme et sa fréquence en HSG est de l'ordre de 5 à $25 \%$. Il existe donc des erreurs ou des imprécisions diagnostiques imputables à l'HSG. Environ $31,5 \%$ des femmes portent des germes banaux dans leurs sécrétions endocervicales. Cinq espèces bactériennes ont été isolées et identifiées dans les sécrétions endocervicales de ces femmes.

$\mathrm{Au}$ nombre de ces espèces, deux (Escherichia coli et Streptocoque D) ont été retrouvés à la fois chez les femmes qui présentent une obstruction tubaire et chez celles qui n'en présentent pas.

Henry-Suchet (1987) avait, par ailleurs, montré qu'il existait une relation entre l'infection et les divers germes aérobies et anaérobies, notamment $E$. coli et Streptocoque D. Trente-cinq virgule un pour cent $(35,1 \%)$ des patientes hébergent dans leurs sécrétions endocervicales les mycoplasmes à un titre pathogène établi par le kit IST2 de bioMérieux. Ce chiffre est plus faible que celui trouvé par Faïhun et Nahoum (1997) $(49,31 \%)$ et par Segonds et al. (1992) (65\%). Ces différences seraient probablement attribuables soit à la technique utilisée soit ou au kit utilisé. Ureaplasma urealyticum a été l'espèce de mycoplasme la plus identifiée, soit 30 cas sur $39(76,9 \%)$. Nos résultats sont proches de ceux de Segonds et al. (1992) qui avaient trouvé $74 \%$. Ce germe a été observé aussi bien chez les femmes ne présentant pas une obstruction tubaire que chez celles qui en présentent mais avec une fréquence plus élevée chez ces dernières. Mycoplasma hominis quant à lui, a été isolé dans $2,56 \%$ des cas. Le taux de l'infection à Chlamydia trachomatis dans cette population d'étude est de $38,7 \%$. L'étude réalisée par Coco et Koonou (2005) et celle de Bezian et al. (1992) ont donné respectivement $20,9 \%$ et $32,4 \%$. Ce dernier résultat est plus proche de celui de notre étude. Par contre, les travaux réalisés par 
Bankolé et al. (2001) en Côte d'Ivoire, ont donné 10,8\%. Muteganya et al. (1997) avaient isolé $C$. trachomatis dans $71 \%$ des cas au niveau de l'endocol. Ces différences pourraient être dues à la population étudiée, à la technique utilisée et au lieu où les prélèvements ont été effectués.

La sérologie chlamydienne est positive chez $36,8 \%$ des femmes présentant une obstruction tubaire contre $40,75 \%$ chez les femmes n'en présentant pas. C. trachomatis n'occasionne des dommages au niveau des trompes utérines qu'à la phase chronique de l'infection; les femmes aux trompes non obstruées feraient donc une chlamydiose en phase aiguë ou subaiguë (Picaud et al., 1990). Cela témoigne que cette infection est aussi présente chez les sujets obstrués que chez les non obstrués. Mefane et al. (1988) avaient noté une sérologie chlamydienne positive chez $84 \%$ des femmes présentant une obstruction tubaire. La responsabilité de ce germe dans les obstructions tubaires a été également reconnue par plusieurs auteurs (Keilani et al., 1989; Nahmonovici et Pastorini, 1989; HenrySuchet, 1991 ; Muteganya et al., 1997). Les différences de fréquences observées pourraient s'expliquer par plusieurs facteurs à savoir: la technique de dépistage utilisée, la taille de l'échantillon et les critères de sélection des patientes, le milieu socioéconomique et environnemental, les traitements mal conduits et l'automédication. Le risque relatif calculé est de 3,49. Cela nous permet de déduire que les femmes infectées feraient donc 3 fois plus l'obstruction tubaire que les non infectées. Les patientes ayant été soumises à une prémédication avant 1'HSG représentent 78 cas, soit 70,3\%. Parmi elles, 21 , soit $26,9 \%$ portent encore des germes banaux. Vingt-six patientes $(33,3 \%)$ ont des mycoplasmes dans leurs sécrétions endocervicales. Cela montre que malgré une antibiothérapie sans identification et sans antibiogramme avant l'HSG, les germes sont encore présents chez ces patientes. L'automédication telle que cela se pratique aujourd'hui, est-elle donc efficace?

On ne saurait en être certain. Une étude plus élaborée sur un échantillon plus grand permettrait sans nul doute de l'approfondir.

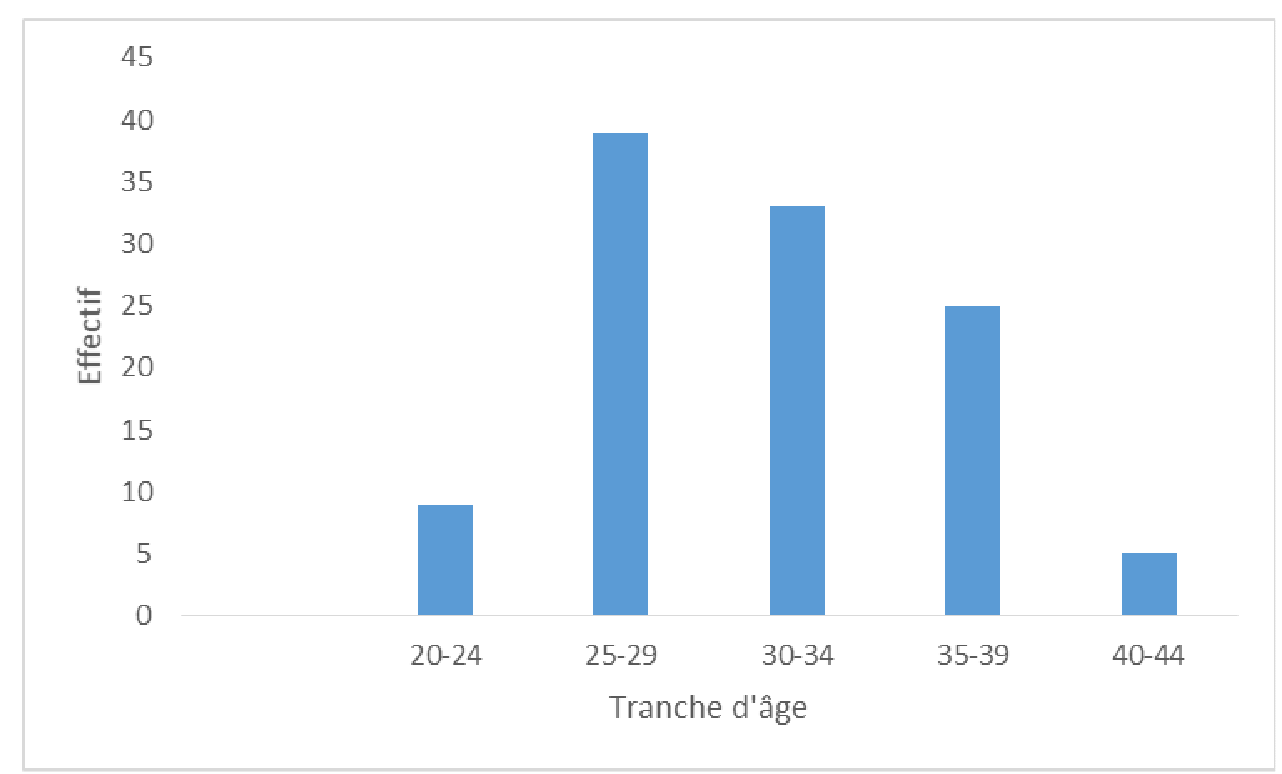

Figure 1: Répartition des patientes par tranche d'âge. 


\section{Conclusion}

Cent onze femmes ont été soumises à des examens radiologiques et bactériologiques. Il a été établi que 51,3\% des femmes admises pour HSG présentent une obstruction tubaire. Elles souffrent d'infections dues aux germes banaux, aux mycoplasmes et à $C$. trachomatis. Le risque relatif calculé a prouvé que les femmes infectées font trois fois plus l'obstruction tubaire que les autres. Face à cette situation, il urge d'informer les femmes sur les divers risques dus aux Infections Sexuellement Transmissibles (IST) en vue de préserver leur fécondité.

\section{REFERENCES}

Attolou A, Hitoemeto C. 2004. Aspects hystérosalpingographiques des obstructions tubaires promixales spasmodiques et intérêt de l'utilisation d'un antispasmodique. Mémoire de Diplôme d'Ingénieur des Travaux (DIT), Université d'Abomey-Calavi, Bénin, p. 70.

Bankolé H, Faye-Kette H, Laruche G, Dabis F, Welfens-Ekra C, Dossou M. 2001. Etude par culture cellulaire de l'infection active due à Chlamydia trachomatis dans une population symptomatique à Abidjan. Bull. Soc. Path. Exot., 94(3): 235-238.

Bezian MC, Pelletier JR, Larousse PH, Dialo B, Bezian JH. 1992. Enquête séroépidémiologique sur les IST à Chlamydia trachomatis en Ethiopie. Bull. Soc. Path. Exot., 85: 125-129.

Bourrit B, Kinkel K. 2007. Comment faire et interpréter une hystérosalpingographie ? Imagerie de la femme, 17(1) : 27-34.

Coco C, Koonou IJ. 2005. Séroprévalence des anticorps anti-chlamydia trachomatis chez les patients consultant dans les services gynécologique et obstétrique de
Cotonou. Mémoire de Diplôme d'Ingénieur des Travaux (DIT), Université d'Abomey-Calavi, Bénin, p. 74.

De Muylder X. 1995. Place de l'hystérosalpingographie dans l'évaluation d'une infertilité en Afrique Noire. Institut de Médecine Tropicale du Service de Santé des Armées, 55(2): 160-164.

Faïhun CFV, Nahoum NH. 1997. Recherche de mycoplasmes dans les écoulements génitaux chez la femme à Cotonou : Technique et fréquence. Mémoire de Diplôme d'Ingénieur des Travaux (DIT), Université d'Abomey-Calavi, Bénin, p.76.

Godjo AH. 1994. La stérilité chez la femme et l'homme : à propos de 364 cas. Thèse de Médecine, Faculté des Sciences de la Santé de Cotonou, Université d'Abomey-Calavi, Bénin, p.96.

Henry-Suchet J. 1991. Place des chlamydioses en gynécologie. La Presse Médicale, 20(1) : 10-12.

Keilani A, Boulieu D, Raudrant D, Carraz M, Quenin P. 1989. Rôle de Chlamydia trachomatis dans les pathologies tubaires (salpingites aiguês et stérilité tubaire): étude microbiologique sur 175 liquides péritonéaux. J. Gynécol. Obstét. Biol. Reprod., 18(2): 167-172.

Lahady R, Daodo H, Razakamaniraka J, Andrianjafimanana $\quad \mathrm{CH} . \quad 2000$. Hystérosalpingographie et stérilité tubaire. M.A.N., 47(5): 252-255.

Mefane C, Benomi D, Nzogue Nguema JP. 1988. L'infection à Chlamydia trachomatis chez la femme à Libreville. M.A.N., 35(9): 647-658.

Muteganya D, Nakintije R, Muhirwa G, Poste B, Ntahonkiriye G, Harerimana S. 1997. Rôle du Chlamydia trachomatis dans les infections utéro-annexielles ou 
leurs séquelles au CHU de Kamenge (Bujumbura-Burundi). M.A.N., 44(1): 69.

Nahmononovici C, Pastorini E. 1989. Infections gynécologiques et stérilité. Rev. Fr. Gynécol. Obstét., 84(3) : 269271.

N'dakena K, Adjamagbo K, Baete S, Hodonou K. 1993. Aspects radiologiques de la pathologie utéro-tubaire dans la stérilité féminine. M.A.N., 40(10) : 650655.
Palmer R, Palmer E. 1975. Les explorations fonctionnelles gynécologiques. $2^{\text {ième }}$ édition. Paris : Masson et $\mathrm{C}^{\mathrm{ie}}, 184-189$.

Picaud A, Berthonneau JP, Nlome-Nze AR, Benoni D, Mefane C, Baud A. 1990. Stérilités tubaires et Chlamydia trachomatis. Corrélations cliniques et microbiologiques. M.A.N., 37(2): 45-49.

Segonds C, Francoual D, Assemekang B. 1992. Mycoplasmes et grossesse : Etude préliminaire. Journal de Gynécologie Obstétrique et Biologie de la Reproduction, 21(4): 385-392. 